\title{
Impacto de la condición corporal sobre la fertilidad en vacas de la provincia de Pastaza- Ecuador
}

Impact of body condition on fertility in cows of the province of Pastaza-Ecuador

\author{
Arias Vaca, M. R. ${ }^{1}$, Pesantez Pacheco J. L. ${ }^{1}$, Lammoglia M. A. ${ }^{2}$, Daniel-Rentería I. ${ }^{2}$, P. R. Marini ${ }^{3,4 \bowtie ~}$ \\ ${ }^{1}$ Universidad de Cuenca - Ecuador. Ecuador. \\ ${ }^{2}$ Facultad de Ciencias Biológicas y Agropecuarias, Universidad Veracruzana, México. \\ ${ }^{3}$ Facultad de Ciencias Veterinarias. \\ ${ }^{4}$ CIC-UNR. Universidad Nacional de Rosario-Argentina \\ ${ }^{\square}$ Autor de correspondencia: pmarini@unr.edu.ar
}

Recibido: $25 / 02 / 18$

Aceptado: 20/05/18

\begin{abstract}
RESUMEN
El objetivo fue evaluar el efecto de la condición corporal sobre la fertilidad en vacas de la provincia de Pastaza-Ecuador. Se utilizaron 2434 registros reproductivos de la base de datos entre el 20092013. Las variables analizadas fueron: estado reproductivo gestante y no gestante, año, raza, condición corporal (CC), celo, toro o pajuela utilizada, técnico inseminador. La muestra para el análisis estadístico de los datos se basó en tres modelos de ANOVA y un modelo mixto. El porcentaje de preñez a nivel provincial logrado con este programa de mejoramiento fue del 60 al 69\%. La CC solo fue significativa para la raza Charolais mestiza, quien mostró un coeficiente de correlación lineal bajo negativo con respecto a la gestación, las otras variables celo, pajuela, técnico tampoco tuvieron diferencias estadísticas $(\mathrm{P}>0.05)$. Pero en el análisis del segundo modelo aplicado se encontró diferencias significativas entre el lugar (cantón) y CC $(\mathrm{P}=0.047)$ y entre la raza de la vaca y raza del toro $(\mathrm{P}=0.006)$. Se concluye que la $(\mathrm{CC})$ influyó sobre la gestación dependiendo del lugar, independiente de la raza de la vaca, factor que no resultó importante en la variable reproductiva estudiada, los factores ambientales: año, cantón y CC, influyen en los niveles de gestación de la población estudiada, de los factores genéticos analizados las razas de la vaca y del padre no influyeron, solamente el factor toro fue importante en la gestación de la vaca y el factor humano resultó determinante como causa de variación en la gestación.
\end{abstract}

Palabras clave: bovinos, ambiente, eficiencia reproductiva, Amazonía

\begin{abstract}
The objective was to evaluate the effect of body condition on fertility in cows from the province of Pastaza-Ecuador. We used 2434 reproductive records from the database between 2009-2013. The variables analyzed were: pregnant and non-pregnant reproductive status, year, race, body condition (CC), heat, bull or straw used, technical inseminator. The sample for the statistical analysis of the data was based on three models of ANOVA and a mixed model. The percentage of pregnancy at the provincial level achieved with this improvement program was 60 to $69 \%$. The CC was only significant for the Charolais mestizo breed, which showed a negative linear correlation coefficient with respect to gestation, the other estrus, straw, and technical variables also did not have statistical differences $(\mathrm{P}>0.05)$. But in the analysis of the second applied model, significant differences were found between the place (canton) and $\mathrm{CC}(\mathrm{P}=0.047)$ and between the breed of the cow and bull
\end{abstract}


breed $(\mathrm{P}=0.006)$. It is concluded that the $(\mathrm{CC})$ influenced the gestation depending on the place, independent of the race of the cow, a factor that was not important in the reproductive variable studied, the environmental factors: year, canton and $\mathrm{CC}$, influence the levels of pregnancy of the studied population, of the genetic factors analyzed, the breeds of the cow and the father did not influence, only the bull factor was important in the gestation of the cow and the human factor was determinant as a cause of variation in the gestation.

Keywords: bovines, environment, reproductive efficiency, amazon.

\section{INTRODUCCIÓN}

El manejo reproductivo en vacas lecheras de alta producción reporta grandes dificultades, debido a que el éxito o el fracaso del mismo están ligados a la incidencia de un gran número de factores a tener en cuenta (raza, condición corporal, ambiente, técnica e inseminador entre otros). Estos factores no ejercen todas las mismas influencias por sí mismos sobre la fertilidad del ganado. Además, en la medida en la que cada uno de estos factores puede ser controlado varía enormemente (Senger, 2005).

López, (2006) mostró que la alteración nutricional y cambios en la condición corporal, tienen un fiel reflejo en los efectos ocasionados a nivel fisiológico y bioquímico, con el fin de mantener los procesos de homeostasis para lo cual se desencadenarían una serie de mecanismos regulados en su gran mayoría por hormonas y otros por compuestos metabólicos. Siendo la nutrición el mayor factor que determina la eficacia reproductiva en ganado de leche, ya que una reducción en la toma de nutrientes, demora el comienzo de la pubertad en novillas Holstein e incrementa el intervalo parto concepción (Ochoa, 2015). Dentro de los factores que afectan la fertilidad, es la consanguinidad. Estudios realizados en Estados Unidos, concluyeron que se ha incrementado la misma en forma dramática desde 1980, hecho que definitivamente se la podría asociar con bajos índices de fertilidad (Revelo, 2013). También el estrés ocasionado por las altas temperaturas, el cual afecta la eficiencia reproductiva de los bovinos en general. Las vacas Bos taurus presentan mayor sensibilidad a los efectos provocados por el calor. Prueba de ello es la baja tasa de fertilidad presente en el verano; cayendo el porcentaje de concepción de $40 \%$ a $15 \%$ (Revelo, 2013). La baja eficacia en la detección de celos limita la fertilidad global del hato. Es un inconveniente que enfrentan todas las explotaciones lecheras que practican inseminación artificial a nivel mundial.También por todos es sabido que existen diferencias significativas entre toros en cuanto a su fertilidad. Al medir el porcentaje de preñez en los primeros servicios, las diferencias oscilan entre el $10 \%$ y el $15 \%$. Además, se ha observado que la fertilidad de los toros oscila entre el $35 \%$ y el $70 \%$. También se observó diferencias en la fertilidad de los distintos toros usando semen heterospérmico (mezcla de semen de más de un toro) (Senger, 2005). En un estudio de campo en el que se compararon un total de 2820 primeros servicios en cuatro rebaños comerciales de Washington, con un total de 11 trabajadores como inseminadores, el más habilidoso obtuvo el $62,7 \%$ de concepción en primer servicio, mientras que el menos habilidoso consiguió solamente el 40,1\%. La variación media entre inseminadores osciló entre el $7 \%$ y el $10 \%$ (Senger, 2005).

Por todo lo anterior expuesto, el objetivo del estudio fue evaluar el efecto de la condición corporal sobre la tasa de preñez de las cuatro 
razas más difundidas en la provincia de Pastaza, bajo un sistema de crianza en la Amazonía Ecuatoriana.

\section{MATERIALES Y MÉTODOS}

Se utilizaron 2434 inseminaciones realizadas durante el periodo 2009 - 2013, que correspondieron al programa de mejoramiento genético impulsado por el Gobierno Descentralizado de Pastaza. Las condiciones climáticas de esta provincia son: Temperatura promedio anual de $25{ }^{\circ} \mathrm{C}$ con un mínimo de $12^{\circ} \mathrm{C}$ y un máximo de $30^{\circ} \mathrm{C}$, con una precipitación de $3500 \mathrm{~mm}$ al año. Humedad media anual del 90 al $100 \%$ y Sus coordenadas son $1^{\circ} 10$ Latitud Sur y $78^{\circ} 10$ de Longitud Oeste; $2^{\circ} 35$ de Latitud Sur y $76^{\circ} 40$ de Longitud Oeste.

\section{Las variables analizadas fueron:}

Variables Independientes: Año (2009, 2010, 2011, 2012, 2013) Raza vaca (Holstein, Browns Swiss, Charolais), Condición corporal (CC) (1 a 5), Celo (Celo Natural CN, Inseminación a Tiempo Fijo IATF), Toro utilizado (Angus rojo; Brown swiss, Charoláis, Holstein negro, Holstein rojo, Jersey, Normando, Simental) e Inseminador (A, B, C, D, E), Cantón (1, 2, 3 y 4)

Variable dependiente: Gestante (gestante, no gestante)

La variable gestación fue analizada asumiendo la distribución teórica de probabilidades binomial y realizando conversión lag y transformación a la distribución normal para cuando se efectuaron los análisis de varianza. Se calcularon las medias estimadas para los factores que resultaron significativos en el análisis de varianza. Se realizó la prueba de comparación post hoc de la DMS. Se generó un modelo utilizando mixtos para evaluar el efecto del toro (aleatorio) dentro del efecto Raza de la vaca, se utilizaron 2355 observaciones. Se excluyeron la raza Normando en el factor Raza de la vaca y solo se emplearon tres cantones, desechando el cantón Arajuno.

Modelo se presenta a continuación:

Yijklmn $=\mu+\mathrm{Ai}+\mathrm{Cj}+\mathrm{Dk}+\mathrm{Hl}+\mathrm{Fm}+\mathrm{Bn}+\mathrm{Sq}+$ eijklmnq

Yijklmn $=$ variable dependiente aleatoria

$\mu=$ constante general

$\mathrm{Ai}=$ efecto del i-ésimo año $(\mathrm{i}=1, \ldots, 4)$

$\mathrm{Cj}=$ efecto de la $\mathrm{j}$-ésima condición corporal $(\mathrm{j}=1, \ldots, 5)$

$\mathrm{Dk}=$ efecto del $\mathrm{k}$-ésimo tipo de celo $(\mathrm{k}=1,2)$

$\mathrm{Hl}=$ efecto del 1 -ésimoinseminador $(\mathrm{l}=1, \ldots, 5)$

$\mathrm{Fm}=$ efecto de la m-ésima raza de la vaca $(\mathrm{m}=1,2,3)$

$\mathrm{Bn}(\mathrm{Fn})=$ efecto de la $\mathrm{n}$-ésima raza del toro $(\mathrm{n}=1, \ldots, 8)$

$\mathrm{Sq}(\mathrm{F}) \mathrm{m}=$ efecto anidado del q-ésimo toro $(\mathrm{q}=1, \ldots, 34)$

en la m-ésima raza de la vaca

eijklmnq $=$ efecto del error

El tercer modelo fue empleado para analizar el factor humano inseminador. Se incluyeron para este análisis todos los cantones, la raza de la vaca, con la raza Normando, el tipo de celo y el Inseminador. No se incluyó el año.

$\mathrm{Se}$ determinaron las frecuencias de los diferentes factores estudiados. Los factores o niveles de un factor que presentaban una frecuencia insuficiente para ser incluidos en los modelos; se realizaron con muestras específicas como el factor inseminador, o como la raza Normando, estos casos fueron analizados utilizando las tablas cruzadas con comparación mediante el estimador Z .

Se estimaron y analizaron los coeficientes de correlación de Pearson y Tau_b de Kendall de forma general y dentro de cada raza de la madre.

\section{RESULTADOS}

La condición corporal en sus seis niveles mostró diferencias significativas $(\mathrm{p} \leq 0,05)$ (Tabla 1). La mayor condición tendría una mejor respuesta en la gestación no fue comprobada. Los niveles de CC 2,50 y 2,75 superaron al de CC 3,00 pero esos tres valores 
de $\mathrm{CC}$ se comportaron igual que la $\mathrm{CC} 2,00 \mathrm{y}$

más baja preñez.

la CC 3,50 y 4,00. La CC 3,00 resultó el de

Tabla 1. Comparaciones entre los niveles de Condición Corporal

\begin{tabular}{ccccc}
\hline & & \multicolumn{2}{c}{ Intervalo de confianza al 95\% } \\
\cline { 4 - 5 } Condición Corporal & Media & Error estándar & DESV EST & CV \\
\hline 2,00 &, $649^{\mathrm{ab}}$ &, 094 &, 97 & 1,49 \\
2,50 &, $666^{\mathrm{b}}$ &, 032 &, 85 & 1,28 \\
2,75 &, $691^{\mathrm{b}}$ &, 039 &, 92 & 1,33 \\
3,00 &, $572^{\mathrm{a}}$ &, 037 &, 97 & 1,69 \\
3,50 &, $719^{\mathrm{ab}}$ &, 046 &, 74 & 1,02 \\
4,00 &, $908^{\mathrm{ab}}$ &, 135 &, 78 & 0,86 \\
\hline
\end{tabular}

Nota: Se basa en la media mínima cuadrática de población modificada.

En la Tabla 2, cada uno de los años analizados mostró que más del $70 \%$ de las vacas presentaban condiciones corporales de 3,00 o menos.

Tabla 2. Distribución de la Condición Corporal en los años estudiados

\begin{tabular}{|c|c|c|c|c|c|c|c|c|}
\hline & \multicolumn{6}{|c|}{ Condición Corporal } & \multirow[b]{2}{*}{ Total } \\
\hline & & 2,00 & 2,50 & 2,75 & 3,00 & 3,50 & 4,00 & \\
\hline \multirow{4}{*}{ Año } & 2010 & 66 & 221 & 182 & 257 & 57 & 12 & 795 \\
\hline & 2011 & 19 & 238 & 164 & 150 & 45 & 5 & 621 \\
\hline & 2012 & 15 & 183 & 174 & 226 & 115 & 13 & 726 \\
\hline & 2013 & 7 & 67 & 39 & 56 & 40 & 4 & 213 \\
\hline \multicolumn{2}{|c|}{ Total } & 107 & 709 & 559 & 689 & 257 & 34 & 2355 \\
\hline
\end{tabular}

Tabla 3. Distribución de la Condición Corporal en los cantones

\begin{tabular}{lcccccccc}
\hline Recuento & & \multicolumn{7}{c}{ Condición Corporal } \\
\cline { 3 - 7 } & & 2,00 & 2,50 & 2,75 & 3,00 & 3,50 & 4,00 & Total \\
\hline \multirow{2}{*}{ Cantón } & Mera & 15 & 142 & 59 & 79 & 14 & 3 & 312 \\
& Pastaza & 92 & 554 & 488 & 501 & 171 & 27 & 1833 \\
& Santa & 0 & 13 & 12 & 109 & 72 & 4 & 210 \\
& Clara & & & & & & \\
Total & & 107 & 709 & 559 & 689 & 257 & 34 & 2355 \\
\hline
\end{tabular}

Solamente en el cantón Santa Clara, la condición corporal presenta puntuaciones de 3,00 o superiores en un porcentaje mayor al $75 \%$. El cantón Pastaza presenta la peor situación.
La Tabla 4 se observa que la raza Charolais presenta algo más de la mitad de sus vacas con condición corporal de 3,00 o más. Las restantes razas presentan más del $60 \%$ de las vacas con condiciones corporales menores de 3,00 . 
Tabla 4. Distribución de la condición corporal en la raza de la vaca

\begin{tabular}{llrrrrrrrr}
\hline & & \multicolumn{7}{c}{ Condición Corporal } & \\
\cline { 3 - 7 } & & 2,00 & 2,50 & 2,75 & 3,00 & 3,50 & 4,00 & Total \\
\hline Raza de la & BS & 33 & 309 & 266 & 344 & 71 & 9 & 1032 \\
vaca & $\mathrm{CH}$ & 12 & 81 & 61 & 97 & 50 & 17 & 318 \\
& $\mathrm{H}$ & 62 & 319 & 232 & 248 & 136 & 8 & 1005 \\
Total & & 107 & 709 & 559 & 689 & 257 & 34 & 2355 \\
\hline
\end{tabular}

En ambos tipos de celo más del 50\% de las vacas presentaban condición corporal inferior al 3,00

Tabla 5 Distribución de la condición Corporal, según, el tipo de celo

\begin{tabular}{ccccc}
\hline & & \multicolumn{2}{c}{ Celo } & \\
\cline { 2 - 4 } & & $\begin{array}{c}\text { Celo } \\
\text { Natural }\end{array}$ & Sincronizado & Total \\
\hline Condición Corporal & 2,00 & 7 & 100 & 107 \\
& 2,50 & 102 & 607 & 709 \\
& 2,75 & 42 & 517 & 559 \\
& 3,00 & 55 & 634 & 689 \\
& 3,50 & 21 & 236 & 257 \\
Total & 4,00 & 4 & 30 & 34 \\
\hline
\end{tabular}

Nota: Condición corporal: Estado de reserva corporal; Sincronizado: AITF.

Solamente en la raza $\mathrm{CH}$ hay una ligera superioridad en la condición corporal (de 3,00 o superior) de las vacas que fueron inseminadas con semen de toros de esa raza. Las demás razas de toros fueron inseminadas en más del $50 \%$ de vacas con condiciones corporales inferiores a 3,00 .

Tabla 6. Distribución de la Condición Corporal en la raza del padre

\begin{tabular}{lllllllll}
\cline { 3 - 7 } & & \multicolumn{3}{c}{ Raza del Padre } & & & & HR \\
\cline { 3 - 8 } & & AR & BS & CH & HN & HR & JE & 1 \\
\hline Condición & 2,00 & 2 & 43 & 11 & 11 & 31 & 2 & 25 \\
& 2,50 & 20 & 294 & 92 & 78 & 118 & 36 & 21 \\
& 2,75 & 9 & 270 & 69 & 56 & 90 & 13 & 11 \\
& 3,00 & 13 & 338 & 106 & 54 & 119 & 15 & 9 \\
Total & 3,50 & 8 & 98 & 53 & 37 & 31 & 12 & 1 \\
& 4,00 & 0 & 10 & 15 & 2 & 4 & 1 & 68 \\
\hline
\end{tabular}

Nota: AR: Angus rojo; BS: Brown swiss; CH: Charoláis; HN: Holstein negro HR: Holstein rojo; JE: Jersey; NR:

Normando

Tabla 7. Análisis de varianza del modelo 


\begin{tabular}{|c|c|c|c|c|c|}
\hline Fuente de Variación & $\begin{array}{l}\text { Suma de } \\
\text { Cuadrados }\end{array}$ & gl & $\begin{array}{r}\text { Cuadrado } \\
\text { Medio } \\
\end{array}$ & Razón-F & $\mathrm{P}<0.05$ \\
\hline cantón & 3.855 & 2 & 1.928 & 8.701 & 0.000 \\
\hline año & 0.185 & 3 & 0.062 & 0.278 & 0.841 \\
\hline condición corporal & 2.497 & 5 & 0.499 & 2.254 & 0.047 \\
\hline tipo de celo & 0.374 & 1 & 0.374 & 1.689 & 0.194 \\
\hline raza del padre & 2.478 & 7 & 0.354 & 1.598 & 0.131 \\
\hline raza de la vaca & 0.191 & 2 & 0.096 & 0.432 & 0.649 \\
\hline \multirow[t]{2}{*}{ raza de la vaca(toro) } & 30.594 & 98 & 0.312 & 1.409 & 0.006 \\
\hline & 492.962 & $\begin{array}{r}222 \\
5\end{array}$ & 0.222 & & \\
\hline
\end{tabular}

Nota:*. La correlación es significativa en el nivel 0,05

El efecto del Cantón, y la Condición corporal fueron significativos $(\mathrm{P}=0.047)$ y el celo no lo fue (0.194), al igual que el efecto del Año que no fue significativo (0.841) entre los efectos no genéticos.

Tampoco los efectos de la raza de la vaca y de la raza del padre fueron significativos (P>0.05). El efecto anidado del Toro dentro de la Raza de la vaca si tuvo alta significación $(\mathrm{P}=0.006)$.

El modelo de la Tabla 8 determinó diferencias significativas para el modelo y la intersección así como también para los efectos del Cantón y del Inseminador. Los restantes efectos no mostraron diferencias significativas como el efecto de la raza de la vaca y la condición corporal

Tabla 8. Análisis de varianza

\begin{tabular}{lccccc}
\hline Origen & $\begin{array}{c}\text { Tipo III de suma de } \\
\text { cuadrados }\end{array}$ & $\mathrm{gl}$ & $\begin{array}{c}\text { Media } \\
\text { cuadrática }\end{array}$ & $\mathrm{F}$ & Sig. \\
\hline Modelo & $8,222^{\mathrm{a}}$ & 15 &, 548 & 2,487 &, 001 \\
corregido & 19,052 & 1 & 19,052 & 86,447 &, 000 \\
Intersección & 4,121 & 3 & 1,374 & 6,233 &, 000 \\
Cantón &, 494 & 3 &, 165 &, 747 &, 524 \\
Raza de la vaca &, 785 & 5 &, 157 &, 712 &, 614 \\
Condición & 2,142 & 4 &, 535 & 2,430 &, 046 \\
corporal & 429,328 & 1948 &, 220 & & \\
Inseminador & 1306,000 & 1964 & & & \\
Error & 437,550 & 1963 & & & \\
Total & & & & & \\
Total corregido & & & & & \\
\hline
\end{tabular}


El inseminador A tiene una media de ,785a, y el $\mathbf{E}$ tiene una media de ,552b por lo cual supera el A supera al B en más de 23 unidades, lo que pone de manifiesto la influencia del factor humano sobre la gestación. No obstante, no se encontraron diferencias significativas entre los inseminadores A, B, C y D. Tampoco se comprobaron diferencias significativas entre los inseminadores $\mathbf{B}, \mathbf{C}, \mathbf{D}$ con el inseminador E.

Los errores estándares no fueron muy altos y solamente los del inseminador con mejor resultado y el de peor resultado y (una cantidad menor de observaciones), hay una ligera superioridad.

Tabla 9. Comparación entre los inseminadores.

\begin{tabular}{|c|c|c|c|c|}
\hline \multirow[b]{2}{*}{ Inseminador } & \multirow[b]{2}{*}{ Media } & \multirow[b]{2}{*}{$\begin{array}{c}\text { Error } \\
\text { estándar }\end{array}$} & \multicolumn{2}{|c|}{$\begin{array}{l}\text { Intervalo de confianza al } \\
95 \%\end{array}$} \\
\hline & & & $\begin{array}{l}\text { Límite } \\
\text { inferior }\end{array}$ & $\begin{array}{c}\text { Límite } \\
\text { superior }\end{array}$ \\
\hline $\mathrm{A}$ &, $785 a$ &, 115 &, 558 & 1,011 \\
\hline B &, $733 a$ & ,067 - - & ,602 & ,864 \\
\hline $\mathrm{C}$ &, $671 a b$ & ,072 &, 530 & 813 \\
\hline D & ,669ab & ,070 & ,532 & 807 \\
\hline $\mathrm{E}$ &, $552 b$ & 139 & ,279 & ,826 \\
\hline
\end{tabular}

Nota: A, B,C,D y E, identificación de los inseminadores.

\section{DISCUSIÓN}

La tendencia de que para una mayor condición corporal habría una mejor respuesta en la gestación no fue comprobado, y este mismo resultado lo tuvo Correa et al, (2013) donde indicó que en vacas Brahman no encontraron influencia de la condición corporal sobre la fertilidad. La mayoría de las vacas se encuentran entre 2,25 y 4,0 mientras que las vacas con $\mathrm{CC}$ inferiores o superiores a este rango generalmente presentan patologías específicas y su calificación es poco relevante (Grigera, 2005), pero en este estudio se obtuvo condiciones corporales muy dispersas donde estuvieron distribuidas así, los niveles de 2,50 y 2,75 superaron al nivel 3,00 pero esos tres niveles se comportaron igual que el nivel 2,00 y los niveles 3,50 y 4,00. Como se observa en el cantón Santa Clara, la condición corporal presenta puntuaciones de 3,00 o superiores en un porcentaje mayor al $75 \%$. El cantón Santa Clara el $95 \%$ o más las ganaderías son de raza Charoláis mestiza o por lo menos tienen esa cruza, ello explica porque existe una coeficiente de correlación lineal bajo, negativo y significativo $(\mathrm{P}<0.05)$, en gran cantidad de los animales con condición corporal de 3 , de ahí también radica la causa de un menor porcentaje de preñez, debido una vez más al estrés calórico en el que se encuentran estos animales.

Un estudio realizado por Correa y Alvarado, (2008), no se encontró un efecto entre la pérdida de condición corporal y los servicios por concepción, parece haber una tendencia inversa, es decir, que a mayor pérdida de condición, menos servicios, esto podría relacionarse con que las vacas con mayor pérdida de condición tienden a tener anestros más prolongados y al reiniciar la actividad ovárica tardíamente, suelen tener menos problemas de fertilidad relacionados con involución uterina e infecciones de tracto reproductor. 
La Tabla 3, mostró marcadas diferencias entre los Cantones. Dentro de la provincia de Pastaza existe varios pisos altitudinales donde están ubicados los diferentes cantones, y por lo cual, hay varios tipos de climas. El cantón Santa Clara, se encuentra a una altura aproximada de $500 \mathrm{msnm}$ con un promedio de temperatura de $30{ }^{\circ} \mathrm{C}$, el cantón Pastaza cuenta con una altitud promedio de $790 \mathrm{msnm}$ y una temperatura entre 20 a $25{ }^{\circ} \mathrm{C}$ y el cantón Mera con una altitud de 1000 msnm y una temperatura promedio de 18 a $20^{\circ} \mathrm{C}$. Esto pudo influir en los resultados reproductivos de los Cantones. La temperatura están influyendo sobre la reproducción y por ende en los índices de fertilidad, nuevamente el estrés calórico arroja claros resultados negativos sobre la fertilidad (Matinez, 2011).

Las vacas Bos taurus presentan mayor sensibilidad a los efectos provocados por el calor. Prueba de ello es la baja tasa de fertilidad presente en el verano; cayendo el porcentaje de concepción de $40 \%$ a $15 \%$ (Revelo, 2013). Se ha observado que los embriones presentan susceptibilidad al estrés calórico, esto disminuye a medida que avanzan en su estado de desarrollo. Por otra parte, el aumento térmico puede afectar el mecanismo de reconocimiento materno de la gestación (Revelo, 2013). Los bovinos que se encuentran en el cantón Santa Clara se encontrarían en estrés calórico permanente.

Gongora y Hernández (2010), encontraron en vacas Holstein Friesian en estro, una disminución de la actividad sexual en el verano frente al invierno (4,5 montas y 8,6 montas, respectivamente); este comportamiento redujo la probabilidad de su detección por observadores entrenados. El porcentaje de celos no detectados en fincas comerciales en Florida (EU) estuvieron entre 76 y $82 \%$ de junio a septiembre y entre 44 y $65 \%$, entre octubre a mayo. Se ha contemplado que una de las principales razones por la que se reduce la fertilidad en la provincia de Pastaza es el estrés calórico que se presenta en los diferentes cantones de la misma y las variaciones de temperatura de cada año.

El celo, fue el único de los factores no genéticos que no fue significativo. Generalmente el celo natural puede ser superior al celo inducido o artificial, pero si se tienen las condiciones adecuadas de manejo y nutrición. El 95\% de los animales son manejados al sogueo, lo cual no permite que los bovinos estén sueltos y expresen las características de un animal en celo natural o próximo a estarlo. Además, se le debe sumar una nutrición desbalanceada, poco tiempo dedicado específicamente para la detección de los celos y el poco entrenamiento de los ganaderos en detectarlos, estos factores hace que se realicen inseminaciones fuera del horario adecuado obteniendo bajo índices de preñez. Rodríguez, (2012), afirma que al realizar la inseminación artificial 6 horas antes o después de la ovulación, la fertilidad tiende a ser más baja. Por lo cual, es importante destacar que, el porcentaje de concepción es mayor cuando la I.A. es realizada antes de la ovulación, lográndose las mejores respuestas entre 7 y 24 horas antes del proceso ovulatorio y una la máxima fertilidad con inseminaciones efectuadas de 13 a 18 horas antes de la ovulación (Rodríguez, 2012).

Las diferencias encontradas entre vacas Holstein Friesian en el reinicio de la actividad ovárica posparto, pueden ser resultado de la adaptación de los grupos raciales a las diferencias en criterios de manejo principalmente el manejo reproductivo y nutricional (Lehmann et al, 2016). A menudo se asocia la mayor producción de leche durante el posparto con un retorno a la ciclicidad más lento y a la fertilidad. Vacas con mayores producciones tienen su primer estro después que aquellas de más baja producción Correa y Alvarado, (2008). 
El factor raza del toro, es un factor muy importante generalmente, pero en la población estudiada no fue un factor determinante. El toro dentro de cada raza como individuo, mostró diferencias importantes; lo cual, indica que se deben analizar las características individuales que ellos tienen con un mayor nivel de detalle y análisis, para tomar decisiones. Dentro de la raza las diferencias encontradas oscilan entre el $10 \%$ y el $15 \%$. Además, se sabe que la fertilidad de los toros oscilaba entre el 35\% y el 70\% (Senger, 2005).

Se encontraron diferencias significativas entre los inseminadores. El factor humano si influenció en los porcentajes de fertilidad, esto se debería a la diferencia en la experiencia en la técnica de inseminación ya que no todos los inseminadores cuentan con el mismo nivel de preparación. Es importante contar con operarios entrenados, capaces de interpretar adecuadamente el comportamiento del animal en celo y que estén capacitados como inseminadores (Wilde, 2005). El factor humano, es de vital importancia en el proceso reproductivo e involucra a todo el personal de la finca, desde el propietario, veterinario hasta el personal de campo. El factor humano debe estar comprometido en detectar adecuadamente los celos, realizar eficientemente los servicios y controlar la calidad del semen.

\section{CONCLUSIÓN}

La condición corporal influyó sobre la preñez, pero fue independiente de la raza de la vaca. Los factores año, cantón, toro e inseminador influyeron en los porcentajes de preñez de la población estudiada.

\section{LITERATURA CITADA}

Correa , E., y Alvarado, J. (2008). Efecto del cambio en la condición corporal, raza y número de partos en el desempeño reproductivo de vacas lecheras.
Agronomía Mesoamericana, 19: 253255. https://doi.org/10.15517/am.v19

Correa Orozco, A., y Uribe, L. (2013). Factores que afectan la preñez en vacas Brahman sometidas a inseminación artificial a tiempo fijo. Revista MVZ Córdoba, 18: 33173326. https://doi.org/10.21897/rmvz.194

Diaz, R. (2011). Reproductivo posparto en vacas lecheras. Ibarra, Imbabura, Ecuador: Memorias del Sexto Seminario Internacional de Buiatría, 1: 19-21.

Gongora , A., y Hernández , A. (2010). La reproducción de la vaca se afecta por las. Revista U.D.C.A Actualidad \& Divulgación Científica, 2: 142 - 143. https://doi.org/10.31910/rudca.v13.n2.

Grigera Juan . (2005). Evaluación del estado corporal de las vacas lecheras. Cordoba: Consultores Elanco Animal Health, 1: 1-8.

Lehmann, J., Fadel $\dagger$, J., and Mogensen, L. (2016). Effect of calving interval and parity on milk yield per feeding day in Danish commercial dairy herds. Journal of Dairy Science,99: 621-633. https://doi.org/10.3168/jds.2015-9583

López, F. (2006). Relación entre condición corporal y eficiencia. Cauca, Colombia: Universidad del Cauca, 4: 81-83.

López, J. (2006). Definición de condición corporal. Relación entre condición corporal y eficiencia. Cauca, Popayán, Colombia: Universidad del Cauca, 4: 78-79.

Matinez , A. (2011). Estrés calórico en el ganado lechero (Boss taurus) de la raza Holstein. Querétalo, Mexico: Universidad Autónoma de Querétaro Facultad de Ingeniería, 1: 1-136. 
Ochoa , R. (2015). Evaluacion de dos metodos de inseminacion artificaial en la preñez con protocolos de IATF en vaconas Holstein. Cuenca, Azuay, Ecuador, 1: 14-70.

Revelo, G. (2013). Evaluación del desempeño reproductivo del hato lechero de la Hacienda "Sandial" localizada en el cantón Montufar, provincia del Carchi en el período 2011 - 2013. Quito, Pichincha, Ecuador: Universidad San Francisco de Quito, 1: 11-83.

Rodríguez , T. (2012). Momento óptimo de inseminación artificial en celo natural y sincronizado en bovinos. Venezuela Ganadera, 34: 287-288.

Senger, P. L. (2005). Factores de fertilidad en el ganado lechero de alta producción. Planeta semex, 1: 1 - 6 .

Wilde , O. (2005). Manual de inseminación artificial de la hembra bovina. Colombia , Colombia : Departamento de Producción Animal, Facultad de Agronomía y Zootecnia. Colombia, 1: 5-37.

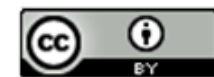

Este tex to está protegido por una licencia licencia Creative Commons 4.0.

\footnotetext{
Usted es libre para Compartir —copiar y redistribuir el material en cualquier medio o form ato-y Adaptar el documento - remezclar transformar y crear a partir del material- para cualquier propósito, incluso para fines comerciales, siempre que cumpla la condición de:

Atribución: Usted debe dar crédito a la obra original de manera adecuada, proporcionar un enlace a la licencia, e in dicar si se han realizado cam bios. Puede hacerlo en cualquier form a razonable, pero no de form a tal que sugiera que tiene el apoyo del licenciante o 10 recibe por el uso que hace de la obra.

Resumendelicencia - Textocompletodelalicencia
} 